\title{
Machine Learning for Masked Face Recognition in COVID-19 Pandemic Situation
}

\author{
Mehreen Fatima $^{1 *}$, Sajjad A. Ghauri ${ }^{1}$, Nooh B. Mohammad ${ }^{2}$, Hannan Adeel $^{1}$, Mubashar Sarfraz $^{3}$ \\ ${ }^{1}$ Department of Electrical Engineering, ISRA University, Islamabad Campus, Pakistan \\ ${ }^{2}$ Department of Computer Science \& Information Systems, American University of Kuwait, Kuwait \\ ${ }^{3}$ Department of Electrical Engineering, NUML, Islamabad, Pakistan
}

Corresponding Author Email: fatimamehreen2000@gmail.com

https://doi.org/10.18280/mmep.090135

Received: 4 August 2021

Accepted: 18 November 2021

\section{Keywords:}

masked face recognition, COVID-19, support

vector machine, random forest

\begin{abstract}
In the current epidemic, the whole world is suffering with the infectious disease i.e., Corona Virus Disease (COVID-19). It is important to wear a mask to minimize the transmission of the disease. When everyone is wearing a face mask, it is difficult for recognition systems to recognize the masked face of a specific person. As some of the facial features are covered behind the mask e.g., mouth and nose. Therefore, the facerecognizing systems are inefficient to recognize the masked faces. To solve this issue, a face recognition system is proposed to recognize masked and unmasked faces. Support vector machine (SVM) and Random Forest (RF) based classifiers are trained on the specific dataset and classifiers effectively recognize the masked and unmasked faces. The classifier recognizes the human facial features such as eyes, eyebrows, forehead, ears, and hair. The dataset is collected in the form of images for 28 classes with and without a face mask. The trained system will recognize the person, whether the person is wearing a mask or not. The recognition accuracy is approximately $98.2 \%$ for different classes and the proposed recognizer is also compared with the state of art existing techniques.
\end{abstract}

\section{INTRODUCTION}

Recognition systems plays a key role in recognizing the person for different purposes. These recognition systems include biometric system, speech recognition system, graphics recognition system, character recognition system, image recognition system and facial recognition system. The biometric system is mainly used in security systems to get access. This system is used to allow the person to access the system by recognizing the texture of their fingers. When the user places a finger on the biometric plate the system matches their fingerprint with the recorded fingerprints in the database. On the other hand, the speech recognition system is used to break the audio of speech recordings into individual voices, to recognize the languages, to fit the most related word in that language and to create the print ready dictation. There are different algorithms used by copying these sounds into the text.

A few decades ago, face recognition was an important topic for the researchers. But it is no longer an important topic for researchers because COVID-19 causes all humans to wear face masks. It is important to wear a face mask to prevent the spread of the virus. However, previous facial recognition systems have failed to recognize the faces in COVID-19 epidemic due to the presence of face masks on human faces. The face mask covers half of the human face. Thus, face recognition systems are not able to recognize the faces properly. To solve this problem, we have proposed a masked face recognition system using two machine learning algorithms i.e., SVM and RF based recognition system. The main objective of the proposed masked face recognition is to efficiently recognize the masked face. The dataset generated are of 28 different class with 1470 number of training and testing images.

In this paper, the SVM and RF based recognizers are used to recognize the masked face images. The recognizer detects the masked image using SURF features and find out the similarities between these features. These features are input to the recognizer structure and the figure of merit is average recognition accuracy. The average recognition accuracy is approximately $97 \%$ with SVM and with the random forest, the average recognition accuracy is $98.2 \%$ for 28 class problem.

The remainder of the paper is organized as follows: Section II presents the comprehensive literature review of existing face recognition techniques. Section III presents the methodology of extraction of various features and the classifier structure. Simulation results are presented with detailed discussion in section IV. Conclusions and future work are finally stated in the section $\mathrm{V}$.

\section{LITERATURE REVIEW}

Generally, when we wear a mask, it creates a problem while recognizing a face. Because it is a difficult facial obstruction as it obstructs most part of facial features which includes the nose. Different techniques have been used to solve this problem. A system is designed to recognize partially occluded faces of human beings. The features of the face can be occluded by mask, glasses, or scarf. The recognition of partial occlusions is done by correlation filters. For recognition of partially excluded faces, mask filters have been developed in Ref. [1] for the identification and security purposes. Histogram 
of oriented gradients (HOG) features in conjunction with SVM have been employed by Dadi and Pillutla [2] also with reduced features using principal component analysis (PCA).

The system is designed to automatically monitor student attendance by recognizing faces [3]. Attendance is being recorded in the system and it is not only useful for one day but can also be accessed in the future. The dataset stores the information of students like their names and faces. The dataset stores students' information like their names and faces. A camera has been fixed in the classroom which marked the attendance by comparing the face of the student sitting in the classroom with the face in the dataset. The algorithm used in the study [4] is for the face recognition using PCA. The system will first identify the person's face by image and then recognize the person. The training image will be compared with the test image to identify the person. The dataset is stored in the form of images. In the principal component analysis, the recognition rate of non-masked faces is much better than that of masked faces.

In Ref. [5], the proposed system is using a deep learning and visual tracking (DLVT) for real-time face recognition. Input image of face is given to the system, the values of all the facial features are calculated and then it will be examined that the input face exists or not. The system proposed by $\mathrm{Li}$ et al. [6] is designed for face recognition using representation-based classification method (RBCM). The two representative techniques used in the system are collaborative representationbased classification (CRC) and linear regression-based classification (LRC). The dataset is compiled from the Internet and contains seven images of each person varying in poses, facial expressions, and light conditions.

The system uses three different types of datasets to identify masked faces. The first is the real-world masked face recognition dataset (RMFRD), which requires real-time identification of the person with and without a face mask. The second is the masked face detector dataset (MFDD) which only detects the mask whether the person is wearing a mask or not. The third one is simulated using masked face recognition dataset (SMFRD) in which the individual is identified but not in real time. Datasets are collected from industry and academia and are freely available to everyone and the algorithm used is multi-granularity [7].

In the study [8], a new corrective algorithm has been introduced which helps in reducing the dimensions of facial images. Facial images are collected from the ORL, YALE and semantic part of the face segment, and the features found in the facial images are extracted from the local ternary pattern and the binary robust invariant scalable key points. Facial images are categorized by deep bailiff network (DBN). Siddiqui et al. [9] designed a system for face detection and recognition which is helpful for security purposes. The system also identifies the age and gender of a person with voice and biometric technology. The dataset stored several images of the person, each with their own unique identity. This system also helps to mark student attendance using machine learning technique.

The dataset is collected in the form of images captured by a camera in Ref. [10]. The different expressions have been captured of the person for the recognition process. The purpose of this procedure is to identify the person in any expression. As proposed by Golwalkar and Mehendale [11], the system is designed to recognize the face of the person wearing the mask. Since it is mandatory to wear a mask in COVID-19 situations, half the face is hidden behind the mask, and it is difficult to identify the person and wearing masks has led to an increase in thefts and robberies. The dataset is stored in the form of photos and live video. The algorithm used in face recognition is the convolution neural network (CNN) with an accuracy of $88.82 \%$. In another article [12], the system is designed for face recognition using $\mathrm{CNN}$. The dataset is in the form of images and when the input icon is given to the system, the system will first check if the given image is stored in the dataset, then proceed to identify the person.

A system is considered for recognizing the human faces which is helpful in marking the present or absent of the human beings [13]. It is also useful for security and identification. However, the algorithm used in the recognition is local binary pattern (LBP). Another system is designed to identify masked faces for the purposes of COVID-19 (Corona virus disease 2019) by Hariri [14]. The system will first cut out the masked face area and then detect facial features from the area of interest using multi-layer perceptron (MLP). The collected dataset is a real-world masked face recognition dataset.

In Ref. [15], datasets are collected from social networks. All datasets of masked faces are first trained using SVM for the application era of video surveillance. As discussed in the study [16], another system is also aimed to recognize the face with a face mask. The method used to detect masked faces is deep and classic machine learning. The algorithms used to detect face masks are decision trees, ensemble and SVM. The dataset is in the form of facial images with and without facial expressions. Some of the existing researches are summarized as:

\begin{tabular}{ccc}
\hline Year & Algorithm & Dataset Collection \\
\hline 2019 & $\begin{array}{c}\text { Deep Learning, CNN and } \\
\text { visual tracking }\end{array}$ & Internet \\
& Principal component & All Information is \\
2020 & analysis (PCA) & collected virtually \\
2020 & Multi-granularity & Industry \& Academia \\
2020 & CNN \& MLP & Real world face \\
2020 & Cascade Classification & Static Camera \\
2021 & CNN & Light Field \\
2021 & Deep Metric Learning & Virtually \\
\hline
\end{tabular}

\section{PROPOSED SYSTEM MODEL}

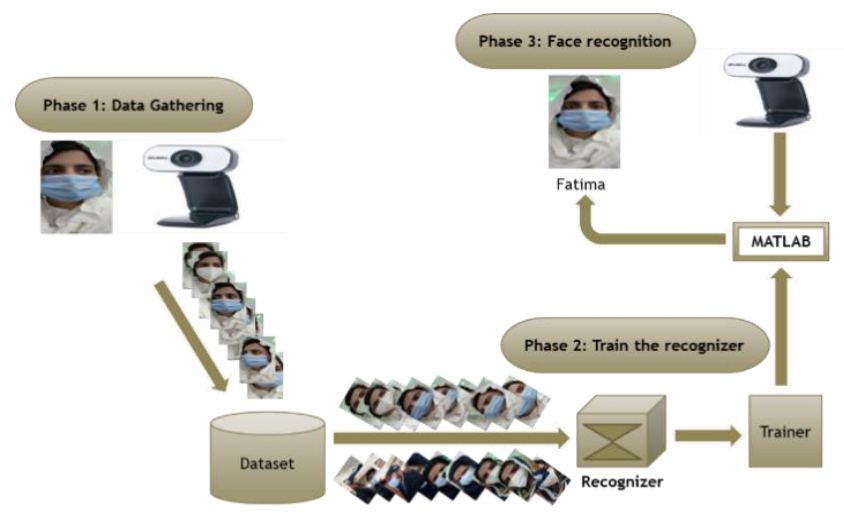

Figure 1. Block diagram of proposed system model

The block diagram of proposed system model is presented in Figure 1. The system model for recognition of masked face is basically categorized into three phases: data collection, training of recognizer and the testing of recognizer. In the first phase, masked face image has been gathered and dataset is 
generated. Secondly, using the dataset, recognizer system is trained by using support vector machine and random forest which is also termed as training of the recognizer. Third phase is the face recognition, which is the last stage and called testing of the classifier.

\subsection{Data set collection}

The data for the recognition problem is gathered in the form of images. Each image is of same size and dimension. Images are captured of a person from different angles. The dataset includes masked and unmasked face images of every person. The collected data set contains 1470 images for the recognizer training and testing. All the images are labelled with their name, institution, and profession. The size of the dataset is $800 \times 600 \times 3$ and total number of elements in each image is 1440000 . The dataset consists of 28 classes and precise picture of the dataset is shown in Figure 2. The dataset and their detailed labelling are shown in the Table 1.

\subsection{Feature extraction}

The speeded up robust features (SURF) are extracted from the image dataset. SURF indicates, a speedy and robust algorithm. It is used to represent the similarity and comparison between the different images. The main aim of SURF is to track or recognize the object.

In the proposed system, SURF is used to recognize the features of human faces and then compare the similarities between the different images. After the extraction, the clustering is used to segregate images having similar features.

1. Choose the number of classes which are used to initialize the center points.

2. The center points are of the same length and known as vectors.

3. The distance is computed between the point and the center of each image.

4. The result is determined based on the closest center.

5. All the above steps are repeated for several iterations, finally, the results are presented.
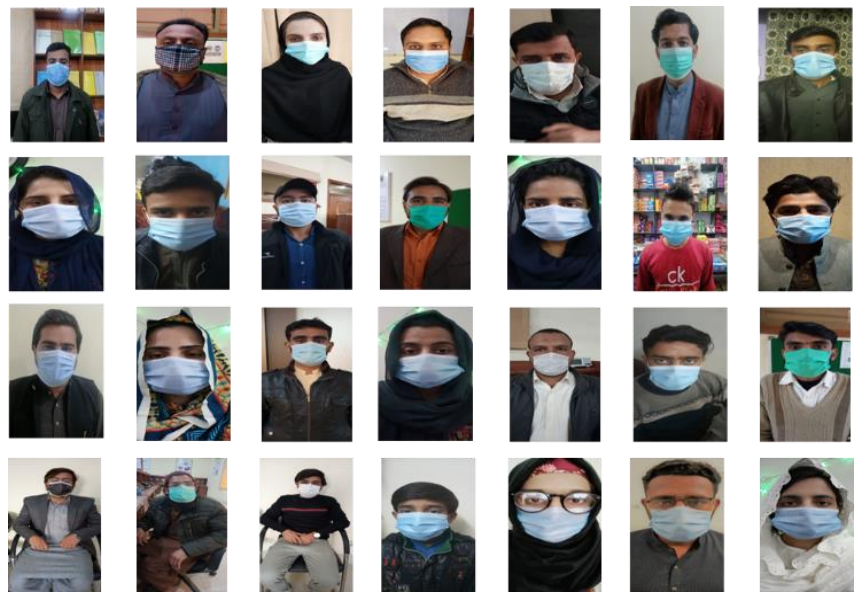

Figure 2. Dataset for masked face recognition

\subsection{Recognizer algorithm}

In this research, support vector machine and random forest are employed as a recognizer, to recognize the masked face. The recognizer algorithm is discussed as following:

Table 1. Recognition dataset with class labelling

\begin{tabular}{|c|c|c|c|c|}
\hline \multicolumn{3}{|c|}{ Image Category with Labels } & \multirow{2}{*}{ Class } & \multirow{2}{*}{ Number of Images } \\
\hline Name & Institution & Profession & & \\
\hline Abubakar & SaveMart & Shopkeeper & $\mathrm{C} 1$ & 60 \\
\hline Ali & PST & Administrator & $\mathrm{C} 2$ & 30 \\
\hline Amna & PIDE & Student & $\mathrm{C} 3$ & 60 \\
\hline Aqeel & PST & Student & $\mathrm{C} 4$ & 60 \\
\hline Arif & SEAS & Student & $\mathrm{C} 5$ & 60 \\
\hline Bilal & SEAS & Librarian & C6 & 30 \\
\hline Binyameen & SEAS & Student & $\mathrm{C} 7$ & 60 \\
\hline EngrAdnan & SEAS & Lecturer & $\mathrm{C} 8$ & 30 \\
\hline EngrSara & SEAS & Lab Engineer & C9 & 60 \\
\hline Gulfam & $\mathrm{PC}$ & Student & $\mathrm{C} 10$ & 60 \\
\hline Gulsham & CS Lab & Lab Attendant & $\mathrm{C} 11$ & 60 \\
\hline Hajra & EOBI & $\mathrm{AD}$ & $\mathrm{C} 12$ & 60 \\
\hline Hasnain & Physics Lab & Lab Attendant & $\mathrm{C} 13$ & 30 \\
\hline Hassan & Preston University & Student & C14 & 60 \\
\hline Iqra & IIU & Student & $\mathrm{C} 15$ & 60 \\
\hline Jhanzaib & EDC Lab & Lab Attendant & C16 & 30 \\
\hline Kashif & SEAS & Lecturer & $\mathrm{C} 17$ & 60 \\
\hline Maryam & Arid University & Student & C18 & 60 \\
\hline Mehreen & SEAS & Student & C19 & 60 \\
\hline Mustajab & IMCB & Student & $\mathrm{C} 20$ & 60 \\
\hline Rafaqat & SEAS & Student & $\mathrm{C} 21$ & 60 \\
\hline Rahid & Robotics Lab & Lab Attendant & $\mathrm{C} 22$ & 30 \\
\hline Rubab & Eden School & Teacher & $\mathrm{C} 23$ & 60 \\
\hline Shehryar & SEAS & Incharge Assistant & $\mathrm{C} 24$ & 30 \\
\hline Turab & IMCB & Student & $\mathrm{C} 25$ & 60 \\
\hline Wahab & Signal Lab & Lab Attendant & $\mathrm{C} 26$ & 60 \\
\hline Waheed & SEAS & Peon & $\mathrm{C} 27$ & 60 \\
\hline Waqar & SEAS & Student & $\mathrm{C} 28$ & 60 \\
\hline
\end{tabular}




\subsubsection{SVM based recognizer}

SVM is a machine learning algorithm which is used for the recognition/classification purposes. A technique used in the SVM algorithm known as kernel trick. It is a function that converts low dimensional space into a high dimensional space. It separates the dataset according to the labels and then gives an output after recognizing the face. The SVMs are used to extract the features from the image by using kernel tricks [17]. The kernel tricks $\left(\mathrm{K}_{\mathrm{n}}\right)$ are used to find out the similarities $\left(\mathrm{S}_{\mathrm{n}}\right)$ between the image given to the system and the image stored in the dataset as shown in the Figure 3. According to these similarities the system will recognize the masked person. The block diagram of the proposed SVM based recognizer is shown in Figure 4.

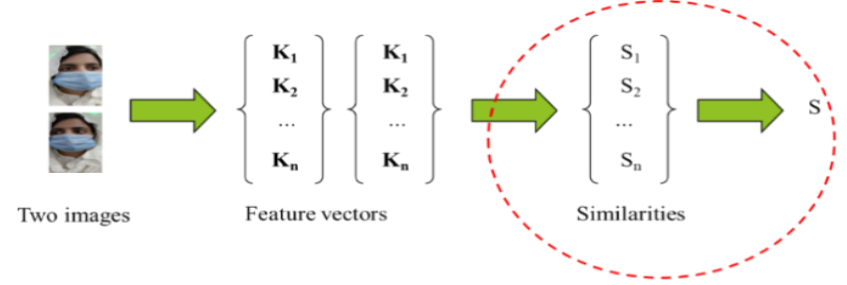

Figure 3. Mathematical model of SVM based recognizer

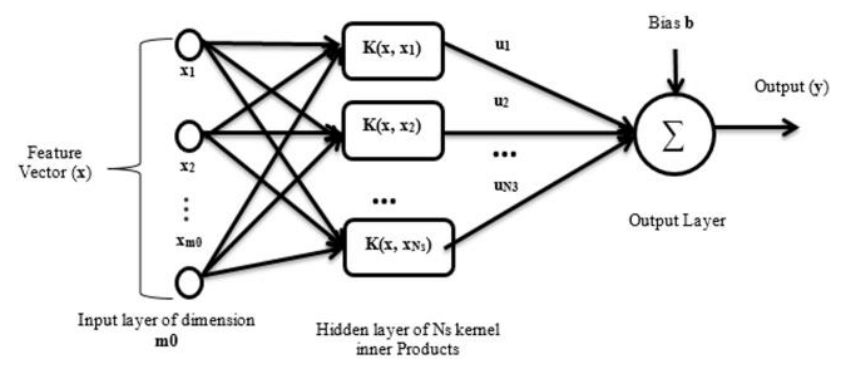

Figure 4. Block diagram of SVM based recognizer

A face recognition system is proposed to recognize faces with and without the face masks. To solve the masked face recognition issues, we have used SVM algorithm. There are two stages of the SVM algorithm. In the first stage, the images are collected from the gallery and reading all the features of each face using SVM. However, the system is trained using SVM and results are stored in the dataset. The second is the testing stage in which the features will be tested using the classifier. The first step in SVM is to import and explore the dataset. After the dataset is pre-processed and split into two parts i.e., attributes and labels. The pre-processed and split data is divided into training and testing sets. Furthermore, predictions are made after training the SVM algorithm. The results are finalized with the help of predictions.

Kernel trick " $\mathrm{K}$ " is a dot product between two functions. The kernel SVM maximizes the hyper plane margin and the transformation formed by it is higher in dimensional space. Let the dataset is $\boldsymbol{X}=\left\{\left(\boldsymbol{x}_{\mathbf{1}}, \boldsymbol{d}_{\mathbf{1}}\right)\left(\boldsymbol{x}_{\mathbf{2}}, \boldsymbol{d}_{\mathbf{2}}\right) \ldots \ldots\left(\boldsymbol{x}_{\boldsymbol{m}}, \boldsymbol{d}_{\boldsymbol{m}}\right)\right\}$, where $\boldsymbol{x}_{\boldsymbol{i}} \varepsilon \mathcal{R}^{n}$ and $\boldsymbol{d}_{\boldsymbol{i}} \boldsymbol{\varepsilon}\{-\mathbf{1},+\mathbf{1}\}, x_{i}$ and $d_{i}$ are representing the data points which are of class type. The optimal hyper plane of SVM is as follows:

$$
\begin{array}{ll}
\mathrm{w}^{\mathrm{T}} \mathrm{x}_{\mathrm{i}}-\mathrm{b} \geq+1, & \mathrm{~d}_{\mathrm{i}}=+1 \\
\mathrm{w}^{\mathrm{T}} \mathrm{x}_{\mathrm{i}}-\mathrm{b} \leq-1, & \mathrm{~d}_{\mathrm{i}}=-1
\end{array}
$$

where, the weight coefficients of the hyper plane are represented by $w$ and $b$ represents the bias network. The minimization problem is as follows: $\min _{\mathrm{w}, \mathrm{b}} \frac{1}{2}\|\mathrm{w}\|^{2}$ subjected to constraints $d_{i}\left(w^{T} x_{i}-b\right) \geq 1$. The optimization problem solved by Lagrangian dual and dual problem is given below:

$$
\begin{aligned}
\min _{w, b} L(w, b, v) & =\frac{1}{2}\|w\|^{2}-\sum_{i=1}^{m} v_{i}\left[d_{i}\left(w^{T} x_{i}-b\right)\right. \\
& -1]
\end{aligned}
$$

After the quadratic programming is applied, the $v_{i}$ values and decision of the class is:

$$
f(x)=\operatorname{sign}\left(w^{T} x-b\right)=\operatorname{sign}\left\{\sum_{i=1}^{m} v_{i} d_{i} x_{i}{ }^{T} x_{i}-b\right\}
$$

which corresponds the non-zeros $v_{i}$ are support vectors. The pseudo code of the proposed recognizer based on SVM is given in Table 2 and Table 3.

Table 2. Extract features from the DATASET

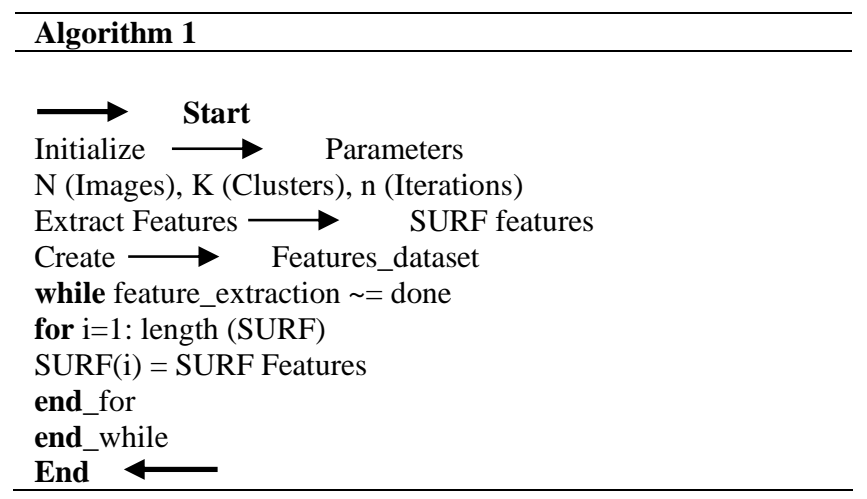

Table 3. SVM recognizer

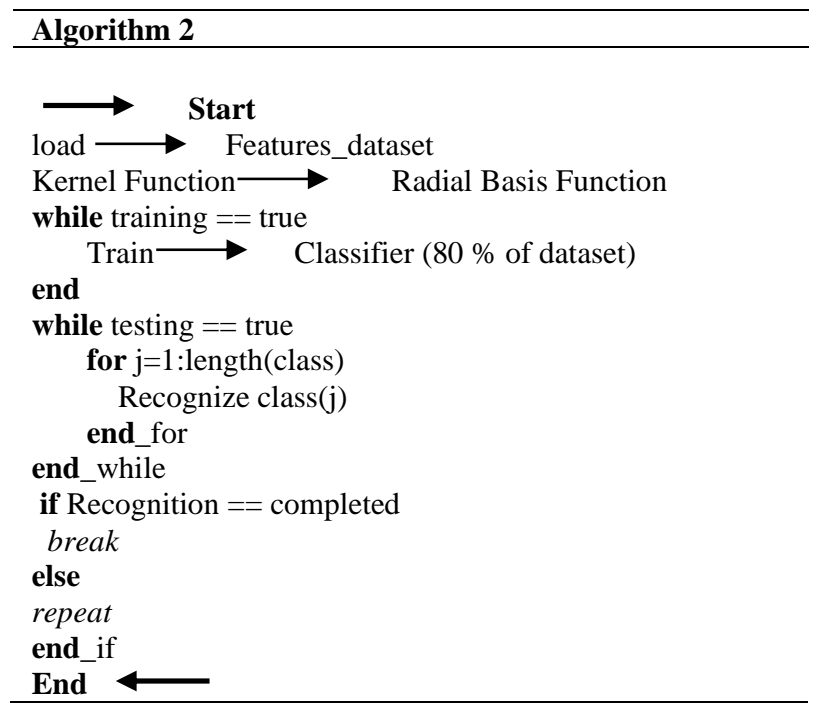

\subsubsection{Random forest based recognizer}

Random forest is an algorithm which is used for the machine learning processes. As the name shown, i.e., random forest, that the forest is made up of the trees. Therefore, the samples are taken from the dataset and then form a tree which predicts the result. The result is finalized based on most voted prediction. Figure 5 depicts the flow of random forest algorithm according to the proposed system. In the first step, 
each image from the dataset is taken as a sample for training. Secondly, for each sample the algorithm will construct a decision tree. From each decision tree we will get the prediction result. Next, from every predicted result, the voting will be made. Lastly, the final predicted result is selected from the most voted prediction.

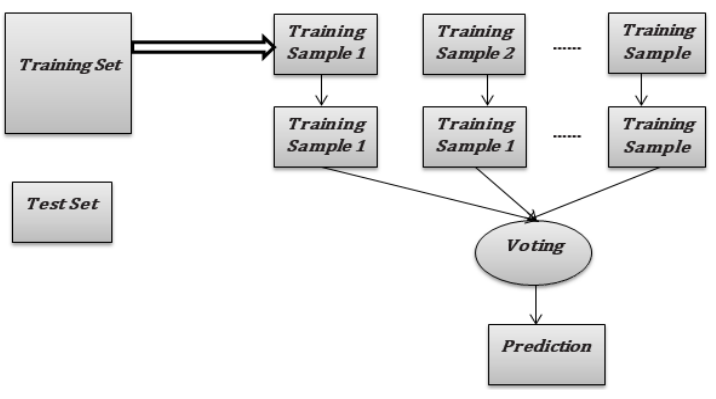

Figure 5. Generalized random forest for face recognition

The start of random forest is to select features $k$ from total number of features $m$. Therefore, it is observed that the features and observations are taken randomly in the image. In the second step, using the best split point, the randomly selected $k$ features are used to find the root node. Thirdly, the daughter nodes are calculated using the best split point. From first three steps the tree with a root node is formed and gets the target as the leaf node. Lastly, 1-4 steps are repeated to randomly create $n$ number of trees. The trees created randomly formed the random forest. In random forest algorithm, the generalization error is presented in the equation given below:

$$
G_{e}=\rho_{x, y}(\operatorname{mar}(x, y)<0)
$$

where, the generalized error is represented by $G_{e}$, the landslide conditioning factors are $x, y$ which indicates over the $x \& y$ space with the margin function mar. Therefore, the margin function mar is shown in the equation below:

$$
\begin{aligned}
\operatorname{mar}(x, y)= & \mathrm{âV}_{m} \mathrm{~J}\left(\mathrm{~h}_{m}(x)=y\right) \\
& -\max _{i \neq j} \mathrm{a} \mathrm{V}_{m} \mathrm{~J}\left(\mathrm{~h}_{m}(x)=i\right)
\end{aligned}
$$

where, $\mathrm{h}_{\mathrm{m}}$ are the classifiers and $\mathrm{J}($.$) is the indicator function.$ The steps for masked face recognition using random forest are given in Table 4.

Table 4. RF based recognizer

Algorithm 3
$\begin{aligned} & \text { load } \\ & \text { 1. Start }\end{aligned}$ Felect the features $k$ randomly from total features $m$.
i. Where $k<m$
2. From the features $k$, node $d$ is calculated using best split
point.
3. Using best split point split the node $d$ into daughter
nodes.
4. Repeat the steps $1-3$ until the 1 number of nodes reached.
5. By repeating the steps $1-4$ build the forest for $n$ times to
create $n$ number of trees.
if Recognition==completed
break
else
repeat
end_if
End

\section{RESULTS AND DISCUSSIONS}

In this section, the proposed algorithms for masked face recognition are simulated on Matlab. The figure of merit of the problem is average recognition accuracy (ARA). The different class problem has been considered in this research. The simulation results are also compared at the end of this section with the state of art existing techniques. The simulation parameters are shown in Table 5. In Figure 6, the two images of a person are shown in which there are lines which indicates the matching of the interested points. The detector is detecting the interested points in the image and constructs the vectors. The interested points of first image will be matched with the other image. If these points are matched of both images, then the SURF features will provide the desired result. The flow chart to recognize the masked face using SVM and RF is shown in Figure 7.

Table 5. Simulation parameters

\begin{tabular}{cc}
\hline Parameters & Value \\
\hline Number of Classes & 29 \\
Image Dataset & 1470 \\
Training of Recognizer & $80 \%$ \\
Testing of Recognizer & $20 \%$ \\
Features & SURF \\
Kernel Function for SVM & Radial Basis Function \\
Max Gini Impurity & 0.1 \\
Number of Trees & 200 \\
Bagging Sample Size & 150 \\
Number of Random Features & 6 \\
\hline
\end{tabular}

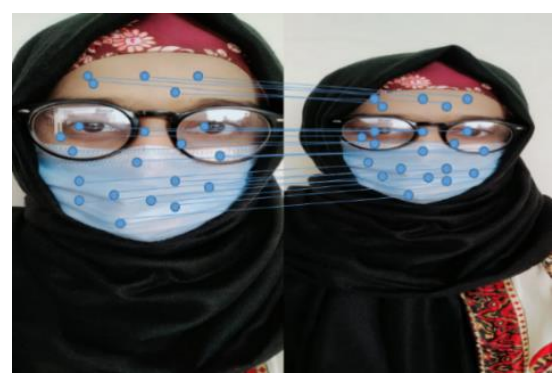

Figure 6. Features matching using SURF features

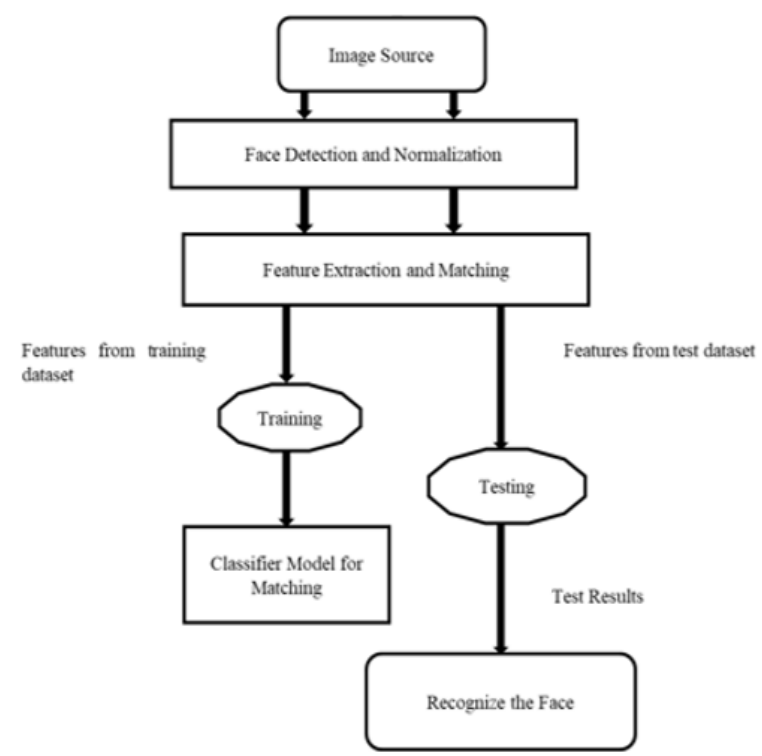

Figure 7. Flow chart of proposed face recognizer 


\subsection{Case I: 4-class problem (SVM)}

The 4-class problem for masked face recognition is considered with four image category IC1, IC2, IC 3 and IC4. The 161328 number of SURF features are extracted from the 4-class dataset with 240 images. Using the K-means clustering, 5000 clusters have been made and number of distinct features selected are 51256. From the confusion matrix shown in Table 6, the SVM recognized all the masked face accurately with $100 \%$ average recognition accuracy.

Table 6. Confusion matrix for 4-class problem (SVM)

\begin{tabular}{ccccc}
\hline ARA & IC1 & IC2 & IC3 & IC4 \\
\hline IC1 & $\mathbf{1 . 0 0}$ & 0.00 & 0.00 & 0.00 \\
IC2 & 0.00 & 1.00 & 0.00 & 0.00 \\
IC3 & 0.00 & 0.00 & $\mathbf{1 . 0 0}$ & 0.00 \\
IC4 & 0.00 & 0.00 & 0.00 & $\mathbf{1 . 0 0}$ \\
\hline
\end{tabular}

\subsection{Case II: 6-class problem (SVM)}

The 6-class problem is considered in the second case for recognition of masked faces based on support vector machine. For the 6-class problem, 195568 features are extracted from the 264 images of dataset. The number of features selected by $\mathrm{K}$-mean clustering algorithm are 65568 . The confusion matrix to show the supremacy of SVM based recognizer for 6-class problem is shown in Table 7. All image categories are recognized accurately with average recognition accuracy of $100 \%$.

Table 7. Confusion Matrix for 6-Class Problem (SVM)

\begin{tabular}{l}
\hline ARA IC1 IC2 IC3 IC4 IC5 IC6 \\
\hline IC1 1.000.000.000.000.000.00 \\
IC2 0.001 .000 .000 .000 .000 .00 \\
IC3 0.000 .001 .000 .000 .000 .00 \\
IC4 0.000 .000 .001 .000 .000 .00 \\
IC5 0.000 .000 .000 .001 .000 .00 \\
IC6 0.000 .000 .000 .000 .001 .00 \\
\hline
\end{tabular}

\subsection{Case III: 6-class problem (Random Forest)}

For random forest algorithm-based recognition 6 image categories are considered. Table 8 presents the 6-class recognition problem confusion matrix. The average recognition accuracy is $99 \%$ and all the classes are exactly recognized with low error rate. As compared with the SVM based recognizer, the SVM performs better than the random forest-based recognizer.

Table 8. Confusion matrix for 6-class problem (RF)

\begin{tabular}{ccccccc}
\hline ARA & IC1 & IC2 & IC3 & IC4 & IC5 & IC6 \\
\hline IC1 & $\mathbf{0 . 9 8}$ & 0.00 & 0.00 & 0.00 & 0.00 & 0.00 \\
IC2 & 0.00 & $\mathbf{0 . 9 9}$ & 0.00 & 0.00 & 0.00 & 0.00 \\
IC3 & 0.00 & 0.00 & $\mathbf{0 . 9 7}$ & 0.00 & 0.00 & 0.00 \\
IC4 & 0.00 & 0.00 & 0.00 & $\mathbf{0 . 9 9}$ & 0.00 & 0.00 \\
IC5 & 0.00 & 0.00 & 0.00 & 0.00 & $\mathbf{0 . 9 8}$ & 0.00 \\
IC6 & 0.00 & 0.00 & 0.00 & 0.00 & 0.00 & $\mathbf{0 . 9 9}$ \\
\hline
\end{tabular}

\subsection{Case IV: 28-class problem (SVM \& RF)}

The 28-class problem is considered and from the 1470 images, 992007 SURF features have been extracted. K-mean clustering to make $5000 \mathrm{~K}$ clusters and selected number of features are 126411. The average recognition accuracy is around $97 \%$ for 28 -class problem, while $98 \%$ for the RF based recognizer shown in Figure 8.

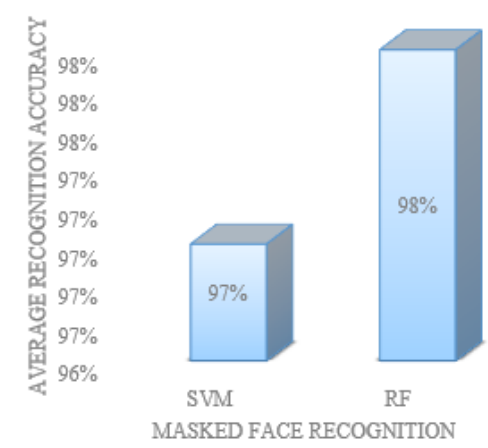

Figure 8. Comparison of ARA for SVM \& RF

\section{CONCLUSION}

The problem occurs in today's COVID-19 situation, where everyone is required to wear a face mask. The security systems are unable to recognize the face. To solve this problem, we have proposed a masked face recognition system. To implement our system in a real-world environment, we have used machine learning algorithms such as SVM \& RBF.

The trained system recognizes the person, whether the person is wearing a mask or not. The average recognition accuracy is approximately $97 \%$ with SVM and with the random forest, the average recognition accuracy is $98.2 \%$ for 28 class problem. From the ARA, the proposed system is helpful for providing a secure system to detect masked faces. If it will be used for real world scenarios it gives more secure environment as compared to current security system.

\section{REFERENCES}

[1] He, E.J., Fernandez, J.A., Kumar, B.V., Alkanhal, M. (2016). Masked correlation filters for partially occluded face recognition. In 2016 IEEE International Conference on Acoustics, Speech and Signal Processing (ICASSP), pp. 1293-1297. https://doi.org/10.1109/ICASSP.2016.7471885

[2] Dadi, H.S., Pillutla, G.M. (2016). Improved face recognition rate using HOG features and SVM classifier. IOSR Journal of Electronics and Communication Engineering, 11(4): 34-44. https://doi.org/10.9790/28341104013444

[3] Aadhirai, S., Sowndarya, R., Keerthika, R., Raj, A.S. (2019). Automated attendance monitoring system based on face recognition technique-A need to the present civilization. Journal of Shanghai Jiaotong University, 16(8). https://doi.org/10.1109/ICCIC.2013.6724266

[4] Ejaz, M.S., Islam, M.R., Sifatullah, M., Sarker, A. (2019). Implementation of principal component analysis on masked and non-masked face recognition. In 2019 1st International Conference on Advances in Science, Engineering and Robotics Technology (ICASERT), pp. 1-5. https://doi.org/10.1109/ICASERT.2019.8934543

[5] Lei, Z., Zhang, X., Yang, S., Ren, Z., Akindipe, O.F. (2020). RFR-DLVT: A hybrid method for real-time face 
recognition using deep learning and visual tracking. Enterprise Information Systems, 14(9-10): 1379-1393. https://doi.org/10.1080/17517575.2019.166896

[6] Li, Z.Q., Sun, J., Wu, X.J., Yin, H.F. (2020). Score level fusion in representation-based classification method for face recognition. Journal of Algorithms \& Computational Technology, 14: 1748302620930943. https://doi.org/10.1177/1748302620930943

[7] Wang, Z., Wang, G., Huang, B., Xiong, Z., Hong, Q., Wu, H., Liang, J. (2020). Masked face recognition dataset and application. arXiv preprint arXiv:2003.09093.

[8] Annamalai, P. (2020). Automatic face recognition using enhanced firefly optimization algorithm and deep belief network. International Journal of Intelligent Engineering and $\quad$ Systems, 13(5): 19-28. https://doi.org/10.22266/ijies2020.1031.03

[9] Siddiqui, M.F., Siddique, W.A., Ahmedh, M., Jumani, A.K. (2020). Face detection and recognition system for enhancing security measures using artificial intelligence system. Indian Journal of Science and Technology, 13(9): 1057-1064.

https://doi.org/10.17485/ijst/2020/v13i09/149298

[10] Sepas-Moghaddam, A., Etemad, A., Pereira, F., Correia, P.L. (2021). Capsfield: Light field-based face and expression recognition in the wild using capsule routing. IEEE Transactions on Image Processing, 30: 2627-2642. https://doi.org/10.1109/TIP.2021.3054476

[11] Golwalkar, R., Mehendale, N. (2020). Masked face recognition using deep metric learning and FaceMaskNet-21. Available at SSRN 3731223. https://dx.doi.org/10.2139/ssrn.3731223
[12] Xu, S., Xu, S., Wang, Z., Pu, C., Meng, Q. (2020). Face Recognition algorithm based on convolutional neural network. In Journal of Physics: Conference Series, 1550(2): 022023. https://dx.doi.org/10.1088/17426596/1550/2/022023

[13] Bah, S.M., Ming, F. (2020). An improved face recognition algorithm and its application in attendance management system. Array, 5: 100014. https://doi.org/10.1016/j.array.2019.100014

[14] Hariri, W. (2021). Efficient masked face recognition method during the Covid-19 pandemic. Signal, Image and Video Processing, 1-8. https://doi.org/10.1007/s11760-021-02050-w

[15] Mundial, I.Q., Hassan, M.S.U., Tiwana, M.I., Qureshi, W.S., Alanazi, E. (2020). Towards facial recognition problem in COVID-19 pandemic. In 2020 4rd International Conference on Electrical, Telecommunication and Computer Engineering (ELTICOM), pp. 210-214. https://doi.org/10.1109/ELTICOM50775.2020.9230504

[16] Loey, M., Manogaran, G., Taha, M.H.N., Khalifa, N.E.M. (2021). A hybrid deep transfer learning model with machine learning methods for face mask detection in the era of the COVID-19 pandemic. Measurement, 167: 108288. https://doi.org/10.1016/j.measurement.2020.108288

[17] Ghauri, S.A., Sarfraz, M., Muhammad, N.B., Munir, S. (2020). Genetic algorithm assisted support vector machine for M-QAM classification. Mathematical Modelling of Engineering Problems, 7(3): 441-449. https://doi.org/10.18280/mmep.070315 